\title{
Guía de aplicación sobre geometría fractal
}

\section{JAIRO MARTÍNEZ PÉREZ, MARIA DEL CARMEN SANDOVAL, LUIS ARTURO POLANÍA QUIZA.}

La siguiente, es una propuesta didáctica para dar a conocer aspectos generales de una geometría no usual en nuestro medio y desconocida en muchos casos, como lo es la GEOMETría FRACTAL o geometría de las formas de la naturaleza.

La geometría tradicional, la Euclídea, es la rama de la matemática que se encarga de las propiedades y de las mediciones de elementos tales como puntos, líneas, planos y volúmenes. La Geometría Euclídea, también describe los conjuntos formados por la reunión de los elementos arriba citados, cuyas combinaciones forman figuras o formas especfiticas.

Sin embargo, formas encontradas en la naturaleza tales como montañas, franjas costeras, sistemas hidrográficos, nubes, hojas, árboles, vegetales, copos de nieve, $y$, un sin número de objetos, no son fácilmente descritos por la geometría tradicional.

La Geometría Fractal, provee una descripción y una forma de modelo matemático para las complicadas formas de la naturaleza. Observemos en el siguiente cuadro las diferencias entre las dos geometrías:

\begin{tabular}{|l|l|}
\hline \multicolumn{1}{|c|}{ EUCLIDEA } & \multicolumn{1}{c|}{ FRACTAL } \\
\hline Tradicional (más de 2000 años) & Moderna (aprox. 10 años) \\
Dimensión entera & Cimensión fraccionaria \\
Trata objetos hechos por el hombre & Apropiada par a formas naturales \\
Descrita por fórmula & Algoritmo recursivo (iteración) \\
\hline
\end{tabular}

* Docentes Universidad Surcolombiana. 
La guía mostrará una cualidad muy importante de los fractales, como lo es la autosimilaridad, que significa que una porción de la figura es semejante a la misma, a gran escala.

Lo anterior se ilustra a través de una guía de aplicación didáctica sobre la construcción de algunas figuras fractales simples.

\section{Construcclón geométrica de algunos fractales simples}

Al elaborar esta guía se plantean los siguientes objetivos:

- Revivir el interés por la geometría.

- Introducir conceptos básicos de transformación y de recursión.

- Realizar actividades de índole geométrico-experlmental, donde se vivencie la geometría fractal.

- Construir geométricamente fractales sencillos, a partir de movimientos de un segmento en el plano.

Obsérvese la siguiente figura:

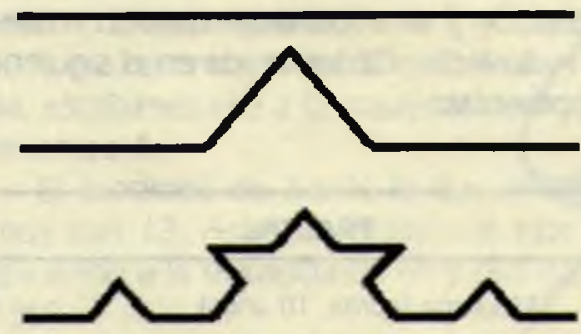

Examinando cuidadosamente las figuras, se descubre que cada figura se construye usando como base la anterior, transformándola de alguna manera.

Observe el paso de la primera figura a la segunda. Considere, que este segundo paso es el resultado de una transformación del segmento de recta, que es la primera figura, en un conjunto de cuatro segmentos de recta. Ahora, si usted observa la tercera figura, verá que ésta se obtiene de la segunda, efectuando la misma transformación a cada uno de los cuatro segmentos que la componen. La figura correspondiente al siguiente paso, es producto de hacer este mismo proceso de transformación a cada uno de los segmentos de recta que componen la tercera figura.

¿Puede usted imaginarse cómo sería la siguiente figura? ¿Podría dibujarla? Si usted sigue y sigue dibujando figuras cada vez con más divisiones del segmento anterior, llegará un momento en el que la longitud de cada línea va a ser muy pequeña, pero la longitud de toda la figura, en sí, es infinita. El límite al que tienden estas figuras de líneas cada vez más pequeñas, es llamada una figura fractal.

Figuras como las anteriores, se pueden obtener a partir de la interpretación de reglas o pasos, aplicados a un segmento de recta dibujado en el plano.

\section{Primera figura fractal}

Consideremos una tortuga cuyo movimiento en el plano se dará según unas instrucciones. La trayectoria la dibujará al dejar tinta regada por cada sitio que pasa. Se utilizarán algunos símbolos como $\mathbf{A}+\mathbf{y}-$; cuya interpretación será:

A : Dibuja una línea recta de una unidad hacia delante.

+ : Gira $60^{\circ}$ a la derecha.

- : Gira $60^{\circ}$ a la izquierda. 


\section{3}

Paideia Surcolombiana

Las instrucciones del recorrido se dan a partir de las siguientes reglas:

$$
R_{1}: A \rightarrow A-A++A-A
$$

Donde $A \rightarrow$, significa que hay que aplicar $\mathbf{R}$, a todas y cada una de las $\boldsymbol{A}$ que aparecen en la hilera.

De esta forma $R_{1}$ sería:

- Muévase una unidad

- Gire $60^{\circ}$ a la izquierda

- Muévase una unidad

- Gire $60^{\circ}$ a la derecha

- Gire $60^{\circ}$ a la derecha

- Muévase una unidad

- Gire $60^{\circ}$ a la izquierda

- Muévase una unidad

Lo cual da la siguiente figura:
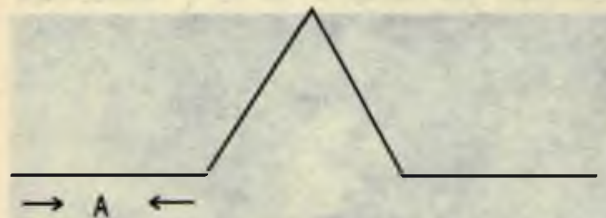

$R_{2}: A-A++A-A-A-A++A-A$ $++A-A++A-A-A-A++A-A$.

Donde esta $\mathbf{A}$, corresponde a un tercio de la $A$ inicial, obteniéndose la siguiente figura:

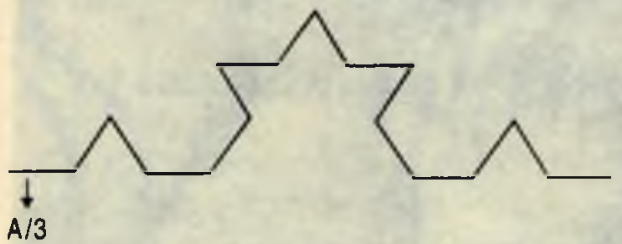

\section{Segunda figura fractal}

Consideremos ahora, una hormiguita cuyo movimiento se realiza según la siguiente regla:
$R_{1}: A \rightarrow A-A+A+A A+A-A+A$

Para ella, el significado de los símbolos es:

A: dibuja una línea de una unidad hacia delante.

+ : girar $90^{\circ}$ a la derecha.

- : girar $90^{\circ}$ a la izquierda.

Obteniéndose el siguiente dibujo:

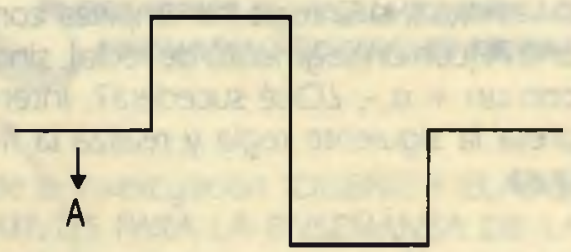

Para la segunda regla y en general, se debe tener en cuenta que una A equivale a aplicar la regla uno $\left(R_{1}\right)$ a cada una de las $A$ que aparecen. La segunda regla será:

$$
\begin{aligned}
& R_{2}: A-A+A+A A+A-A+A-A- \\
& A+A+A A+A-A+A+A-A+A+A A+A- \\
& A+A+A-A+A+A A+A-A+A- \\
& A+A+A-A-A+A+A-A+A+A A+A- \\
& A+A-A-A+A+A A+A-A+A+A- \\
& A+A+A A+A-A+A
\end{aligned}
$$

La medida del segmento $A$, en esta segunda transformación, será un cuarto de la original ( $A / 4)$, en la tercera regla $1 / 16$ de la inicial $(A / 16)$, y así sucesivamente. Gráficamente se tiene:

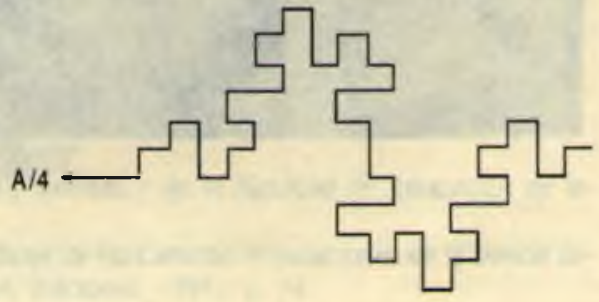




\section{4}

Ĺnıversıdad Surcolombıana

\section{Observaciones}

El resultado al aplicar la regla es una figura fractal, de ahí que la interpretación que se hace de dicha regla debe ser precisa:

- Si se cambia el ángulo, la figura resultante no siempre es la esperada. Dibuja la figura que resulta al aplicar $+: 45^{\circ}$ en cada una de las reglas anteriores ¿Qué observas? ¿Sigue siendo un fractal?

- Ahora, si la regla no empieza con una $\mathbf{A}$ (con un segmento de recta), sino con un + o-, ¿Qué sucederá?. Interpreta la siguiente regla y realiza la figura:

$$
R_{1}: \rightarrow-\mathrm{AA}++\mathrm{AA}++\mathrm{AA}-
$$

Donde:

- : Giro $60^{\circ}$ hacia la izquierda.

+ : Giro de $60^{\circ}$ hacia la derecha.

\section{BIBLIOGRAFÍA}

1. GÓMEZ, Pedro. "MATEBÁSICAMÁTICA". Una empresa docente, Universidad de Los Andes. 1994.

2. SCHROEDER, Manfred. "FRACTALS, CHAOS, POWER LAWS". Editorial K-tdra. Universidad de Maryland.

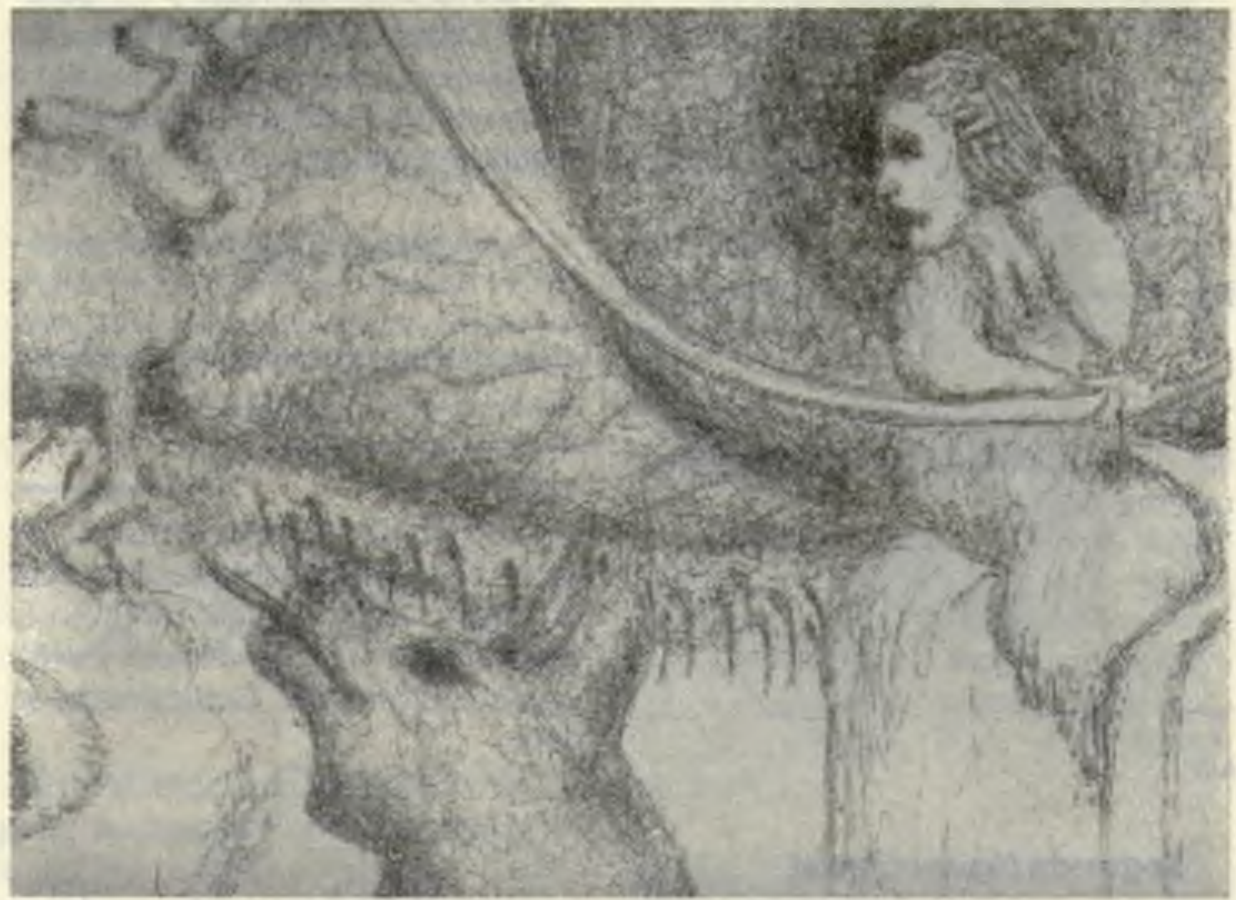

